\title{
Radionuclide therapy in the time of COVID-19
}

\author{
Tessa Brabander $^{1}$ (D) $\cdot$ Hans Hofland ${ }^{2}$
}

Received: 18 May 2020 / Accepted: 7 June 2020 / Published online: 16 June 2020

(C) Springer-Verlag GmbH Germany, part of Springer Nature 2020

Dear Sir,

We read with interest the article by Assidi et al. "Key elements of preparedness for pandemic coronavirus disease 2019 (COVID-19) in nuclear medicine units" [1]. The authors describe general recommendations for nuclear medicine units during COVID-19, including the use of personal protective equipment and management of patients and environmental hygiene.

The authors also recommend to postpone any therapeutic procedure with radiopharmaceuticals according to clinical need. The pandemic of SARS-CoV-2 disease is one of the biggest challenges in the recent history of medicine and the effects on the healthcare systems worldwide will be noticeable for many years. However, treatment of patients with malignant disease remains crucial for their very survival. As our institute is one of the largest peptide receptor radionuclide therapy (PRRT) centres in Europe, providing PRRT for the majority of neuroendocrine tumour patients in The Netherlands, a complete stop of patients for several months could have detrimental health effects for our patients. The COVID-19 epidemic in The Netherlands started on February 27, 2020, with the first documented patient and has since led to 43,481 confirmed infections and 5590 casualties caused by COVID-19 (dated May 14, 2020).

Since the start of the pandemic in The Netherlands, we have continued clinical treatment with 177Lu-DOTATATE, iodine-131 and radioembolization with yttrium-90 for indications of malignant disease. Our revised protocol established on March 16 included calling patients before admission to ask

This article is part of the Topical Collection on Letter to the Editor

Tessa Brabander

t.brabander@erasmusmc.nl

1 Department of Radiology \& Nuclear Medicine, Erasmus Medical Center, Rotterdam, The Netherlands

2 Department of Internal Medicine, Section of Endocrinology, ENETS Center of Excellence, Erasmus Medical Center, Rotterdam, The Netherlands for the existence of respiratory symptoms or fever, in which case treatment would be postponed. Patients arrived at the treatment facility alone and were confined to their individual rooms during the overnight admission. Staff and nurses increased their use of personal protective equipment and environmental hygiene around patients.

Between February 27 and May 14, a total of 107 cycles of 177Lu-DOTATATE was administered to 83 individual patients at our centre. In that same period, 21 patients received iodine-131 and 7 patients underwent yttrium-90 radioembolization. During admission, only one patient developed transient respiratory symptoms and fever after treatment with radioiodine. He was tested SARS-CoV-2 negative on nasal swab and had no COVID-related abnormalities on chest CT. In follow-up, one of our PRRT patients was admitted to the hospital with COVID-19-induced immune thrombocytopenia (ITP), after being infected by his spouse [2]. None of other patients treated with 177Lu-DOTATATE, iodine-131 or yttrium-90 radioembolization developed COVID-19 during follow-up.

Radionuclide therapy has not been shown to increase susceptibility to or complication risk of viral infections. In our previous series of 610 patients receiving PRRT because of advanced neuroendocrine tumours, transient CTCAE grade $3 / 4$ lymphopenia occurred in $50 \%$ but were not accompanied with the occurrence of severe viral infections. In recently emerging data on COVID-19, patients with cancer appear to have an increased risk of worse outcome, particularly in metastatic stages [3]. The additional risk appears limited in patients with thyroid cancer and those treated with radiation therapy.

The possible consequences of postponing therapeutic procedures with radiopharmaceuticals for patients with malignant disease should be individually weighed against the possible risk of a COVID-19 infection during radionuclide therapy. Our experience in a large PRRT centre in The Netherlands demonstrates that it is safe to continue radionuclide therapy amidst the COVID-19 epidemic when using the right precautions. 


\section{Compliance with ethical standards}

Conflict of interest Author TB has received speaker honorarium, research grant and joined advisory boards from Advanced Accelerator Applications. Author JH declares that he has no conflict of interest.

Ethical approval This article does not contain any studies with human participants or animals performed by the authors.

\section{References}

1. Assadi M, Gholamrezanezhad A, Jokar N, Keshavarz M, Picchio M, Seregni E, Bombardieri E, Chiti A. Key elements of preparedness for pandemic coronavirus disease 2019 (COVID-19) in nuclear medicine units. Eur J Nucl Med Mol Imaging. 2020:1-8. https://doi.org/ 10.1007/s00259-020-04780-4.

2. Zulfiqar AA, Lorenzo-Villalba N, Hassler P, Andrès E. Immune Thrombocytopenic Purpura in a Patient with Covid-19. N Engl J Med. 2020;382(18):e43. https://doi.org/10.1056/NEJMc2010472.

3. Dai M, Liu D, Liu M, Zhou F, Li G, Chen Z, et al. Patients with cancer appear more vulnerable to SARS-CoV-2: a multicenter study during the COVID-19 outbreak. Cancer Discov. 2020;10(6):783791. https://doi.org/10.1158/2159-8290.CD-20-0422.

Publisher's note Springer Nature remains neutral with regard to jurisdictional claims in published maps and institutional affiliations. 